\title{
SPECIAL RELATIVITY OVER THE FIELD OF RATIONAL NUMBERS
}

\author{
JUDIT X. MADARÁSZ AND GERGELY SZÉKELY
}

\begin{abstract}
We investigate the question: what structures of numbers (as physical quantities) are suitable to be used in special relativity? The answer to this question depends strongly on the auxiliary assumptions we add to the basic assumptions of special relativity. We show that there is a natural axiom system of special relativity which can be modeled even over the field of rational numbers.
\end{abstract}

\section{INTRODUCTION}

In this paper, we investigate, within an axiomatic framework, the question: what structures of numbers (as physical quantities) are suitable to be used in special relativity? There are several reasons to investigate this kind of questions in the case of any theory of physics. First of all, we cannot experimentally verify whether the structure of quantities is isomorphic to the field of real numbers. Moreover, the fact that the outcome of every measurement is a finite decimal suggests that rational numbers (or even integers) should be enough to model physical quantities. Another reason is that these investigations lead to a deeper understanding of the connection of the mathematical assumptions about the quantities and the other (physical) assumptions of the theory. Hence these investigations lead to a deeper understanding of any theory of physics, which may come handy if we have to change some of the basic assumptions for some reason. For a more general perspective of this research direction, see [4].

So in general we would like to investigate the question

"What structure can numbers have in a certain physical theory?"

To introduce the central concept of our investigation, let Th be a theory of physics that contains the concept of numbers (as physical quantities) together with some algebraic operations on them (or at least these concepts are definable in Th). In this case, we can introduce notation

Date: November 9, 2018.

Key words and phrases. relativity theory, special relativity, rational numbers, axiomatic theories, first-order logic. 
$\operatorname{Num}(\mathrm{Th})$ for the class of the possible quantity structures of theory Th:

$\operatorname{Num}(\mathrm{Th})=\{\mathfrak{Q}: \mathfrak{Q}$ is a structure of quantities

$$
\text { over which Th has a model. }\}
$$

In this paper, we investigate our question only in the case of special relativity. However, this question can be investigated in any other physical theory the same way.

We will see an axiom on observers implying that positive numbers have square roots. Therefore, we recall that Euclidean fields, which got their names after their role in Tarski's first-order logic axiomatization of Euclidean geometry [18], are ordered fields in which positive numbers have square roots.

Our axiom system for $d$-dimensional special relativity $\left(\right.$ SpecRel $_{d}$, see $\mathrm{p}$ 5) captures the kinematics of special relativity perfectly if $d \geq 3$, see

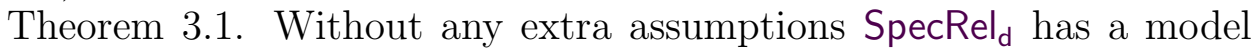
over every ordered field, i.e.,

$$
\operatorname{Num}\left(\text { SpecRel }_{\mathrm{d}}\right)=\{\mathfrak{Q}: \mathfrak{Q} \text { is an ordered field }\},
$$

see Remark 3.3. Therefore, SpecRel has a model over $\mathbb{Q}$ (the field of rational numbers), too. However, if we assume that inertial observes can move with arbitrary speed less than that of light, see AxThExp on $\mathrm{p}[6$, then every positive number has to have a square root if $d \geq 3$ by Theorem 3.2, i.e.,

$$
\operatorname{Num}\left(\text { SpecRel }_{d}+\operatorname{AxThExp}\right)=\{\mathfrak{Q}: \mathfrak{Q} \text { is a Euclidean field }\} \text { if } d \geq 3,
$$

see [4]. In particular, the number structure cannot be the field of rational numbers if AxThExp is assumed and $d \geq 3$, i.e.,

$$
\mathbb{Q} \notin N u m\left(\text { SpecRel }_{d}+\text { AxThExp) if } d \geq 3 .\right.
$$

Theorem 3.4, the main result of this paper, shows that our axiom system SpecRel has a model over $\mathbb{Q}$ (in any dimension) if we assume axiom AxThExp only approximately, i.e.,

$$
\mathbb{Q} \in \operatorname{Num}\left(\text { SpecRel }_{d}+\text { AxThExp }^{-}\right) \text {if } d \geq 2,
$$

see the precise formulation of AxThExp- on p 6. Assuming AxThExp instead of AxThExp is reasonable because we cannot be sure in anything perfectly accurately in physics. Theorem 3.4 implies that SpecRel + AxThExp can be modeled over every subfield of the field of real numbers $(\mathbb{R})$, see Corollary 3.5, and we conjecture that this axiom system has a model over every ordered field, see Conjecture 3.6.

An interesting and related approach of Mike Stannett introduces two structures one for the measurable numbers and one for the theoretical numbers and assumes that the set of measurable numbers is dense in the set of theoretical numbers, see [16].

We chose first-order predicate logic to formulate our axioms because experience (e.g., in geometry and set theory) shows that this logic is the 
best logic for providing an axiomatic foundation for a theory. A further reason for choosing first-order logic is that it is a well defined fragment of natural language with an unambiguous syntax and semantics, which do not depend on set theory. For further reasons, see, e.g., [1, §Why FOL?], [5], [17, §11], [19], [20].

\section{The LANGuage of OUR TheORIES}

To our investigation, we need an axiomatic theory of special relativity. Therefore, we will recall our axiom system SpecRel ${ }_{d}$ in Section 3. To write up any axiom system, we have to choose the set of basic symbols of its language, i.e., what objects and relations between them will be used as basic concepts.

Here we will use the following two-sorted 1 language of first-order logic (FOL) parametrized by a natural number $d \geq 2$ representing the dimension of spacetime:

$$
\{B, Q ; \mathrm{IOb}, \mathrm{Ph},+, \cdot, \leq, \mathrm{W}\},
$$

where $B$ (bodies) and $Q$ (quantities) are the two sorts, IOb (inertial observers) and $\mathrm{Ph}$ (light signals) are one-place relation symbols of sort $B,+$ and $\cdot$ are two-place function symbols of sort $Q, \leq$ is a two-place relation symbol of sort $Q$, and $\mathrm{W}$ (the worldview relation) is a $d+2$ place relation symbol the first two arguments of which are of sort $B$ and the rest are of sort $Q$.

Relations $\operatorname{IOb}(m)$ and $\mathrm{Ph}(p)$ are translated as " $m$ is an inertial observer," and " $p$ is a light signal," respectively. To speak about coordinatization of observers, we translate relation $\mathrm{W}\left(k, b, x_{1}, x_{2}, \ldots, x_{d}\right)$ as "body $k$ coordinatizes body $b$ at space-time location $\left\langle x_{1}, x_{2}, \ldots, x_{d}\right\rangle$," (i.e., at space location $\left\langle x_{2}, \ldots, x_{d}\right\rangle$ and instant $x_{1}$ ).

Quantity terms are the variables of sort $Q$ and what can be built from them by using the two-place operations + and $\cdot$, body terms are only the variables of sort $B \cdot \operatorname{IOb}(m), \operatorname{Ph}(p), \mathrm{W}\left(m, b, x_{1}, \ldots, x_{d}\right), x=y$, and $x \leq y$ where $m, p, b, x, y, x_{1}, \ldots, x_{d}$ are arbitrary terms of the respective sorts are so-called atomic formulas of our first-order logic language. The formulas are built up from these atomic formulas by using the logical connectives not $(\neg)$, and $(\wedge)$, or $(\vee)$, implies $(\rightarrow)$, if-and-only-if $(\leftrightarrow)$ and the quantifiers exists $(\exists)$ and for all $(\forall)$.

To make them easier to read, we omit the outermost universal quantifiers from the formalizations of our axioms, i.e., all the free variables are universally quantified.

We use the notation $Q^{n}$ for the set of all $n$-tuples of elements of $Q$. If $\overline{\mathbf{x}} \in Q^{n}$, we assume that $\overline{\mathbf{x}}=\left\langle x_{1}, \ldots, x_{n}\right\rangle$, i.e., $x_{i}$ denotes the $i$-th

\footnotetext{
${ }^{1}$ That our theory is two-sorted means only that there are two types of basic objects (bodies and quantities) as opposed to, e.g., Zermelo-Fraenkel set theory where there is only one type of basic objects (sets).
} 
component of the $n$-tuple $\overline{\mathbf{x}}$. Specially, we write $\mathbf{W}(m, b, \overline{\mathbf{x}})$ in place of $\mathrm{W}\left(m, b, x_{1}, \ldots, x_{d}\right)$, and we write $\forall \overline{\mathbf{x}}$ in place of $\forall x_{1} \ldots \forall x_{d}$, etc.

We use first-order logic set theory as a meta theory to speak about model theoretical terms, such as models, validity, etc. The models of this language are of the form

$$
\mathfrak{M}=\left\langle B, Q ; \mathrm{IOb}_{\mathfrak{M}}, \mathrm{Ph}_{\mathfrak{M}},+_{\mathfrak{M}}, \cdot_{\mathfrak{M}}, \leq_{\mathfrak{M}}, \mathrm{W}_{\mathfrak{M}}\right\rangle,
$$

where $B$ and $Q$ are nonempty sets, $\mathrm{IOb}_{\mathfrak{M}}$ and $\mathrm{Ph}_{\mathfrak{M}}$ are subsets of $B$, $+_{\mathfrak{M}}$ and $\cdot_{\mathfrak{M}}$ are binary functions and $\leq_{\mathfrak{M}}$ is a binary relation on $Q$, and $\mathrm{W}_{\mathfrak{M}}$ is a subset of $B \times B \times Q^{d}$. Formulas are interpreted in $\mathfrak{M}$ in the usual way. For the precise definition of the syntax and semantics of first-order logic, see, e.g., [7, §1.3], [8, §2.1, §2.2].

\section{Axioms FOR SPECIAL RELATIVITY}

Now having our language fixed, we can recall axiom system SpecRel $_{d}$, as well as two theorems on SpecRel ${ }_{d}$ related to our investigation.

The key axiom of special relativity states that the speed of light is the same in every direction for every inertial observers.

AxPh: For any inertial observer, the speed of light is the same everywhere and in every direction (and it is finite). Furthermore, it is possible to send out a light signal in any direction (existing according to the coordinate system) everywhere:

$$
\begin{aligned}
\operatorname{IOb}(m) \rightarrow \exists c_{m}\left[c_{m}>0 \wedge \forall \overline{\mathbf{x}} \overline{\mathbf{y}}(\exists p[\operatorname{Ph}(p) \wedge \mathrm{W}(m, p, \overline{\mathbf{x}})\right. \\
\left.\left.\wedge \mathrm{W}(m, p, \overline{\mathbf{y}})] \leftrightarrow \operatorname{space}^{2}(\overline{\mathbf{x}}, \overline{\mathbf{y}})=c_{m}^{2} \cdot \operatorname{time}(\overline{\mathbf{x}}, \overline{\mathbf{y}})^{2}\right)\right], 2
\end{aligned}
$$

where $\operatorname{space}^{2}(\overline{\mathbf{x}}, \overline{\mathbf{y}}):=\left(x_{2}-y_{2}\right)^{2}+\ldots+\left(x_{d}-y_{d}\right)^{2}$ and time $(\overline{\mathbf{y}}, \overline{\mathbf{y}}):=$ $x_{1}-y_{1}$.

To get back the intended meaning of axiom $\mathrm{AxPh}$ (or even to be able to define subtraction from addition), we have to assume some properties of numbers.

In our next axiom, we state some basic properties of addition, multiplication and ordering true for real numbers.

AxOField: The quantity part $\langle Q,+, \cdot, \leq\rangle$ is an ordered field, i.e.,

- $\langle Q,+, \cdot\rangle$ is a field in the sense of abstract algebra; and

- the relation $\leq$ is a linear ordering on $Q$ such that

i) $x \leq y \rightarrow x+z \leq y+z$ and

ii) $0 \leq x \wedge 0 \leq y \rightarrow 0 \leq x y$ holds.

Using axiom AxOFiled instead of assuming that the structure of quantities is the field of real numbers not just makes our theory more flexible,

\footnotetext{
${ }^{2}$ That is, if $m$ is an inertial observer, there is a is a positive quantity $c_{m}$ such that for all coordinate points $\overline{\mathbf{x}}$ and $\overline{\mathbf{y}}$ there is a light signal $p$ coordinatized at $\overline{\mathbf{x}}$ and $\overline{\mathbf{y}}$ by observer $m$ if and only if equation $\operatorname{space}^{2}(\overline{\mathbf{x}}, \overline{\mathbf{y}})=c_{m}^{2} \cdot \operatorname{time}(\overline{\mathbf{x}}, \overline{\mathbf{y}})^{2}$ holds.
} 
but also makes it possible to meaningfully investigate our main question. Another reason for using AxOField instead of $\mathbb{R}$ is that we cannot experimentally verify whether the structure of physical quantities are isomorphic to $\mathbb{R}$. Hence the assumption that the structure of quantities is $\mathbb{R}$ cannot be empirically supported. The two properties of real numbers which are the most difficult to defend from empirical point of view are the Archimedean property, see [11], [12, §3.1], [13], [14, and the supremum property 3

We also have to support $\mathrm{AxPh}$ with the assumption that all observers coordinatize the same "external" reality (the same set of events). By the event occurring for observer $m$ at point $\overline{\mathbf{x}}$, we mean the set of bodies $m$ coordinatizes at $\overline{\mathbf{x}}$ :

$$
\mathrm{ev}_{m}(\overline{\mathbf{x}}):=\{b: \mathbf{W}(m, b, \overline{\mathbf{x}})\} .
$$

AxEv: All inertial observers coordinatize the same set of events:

$$
\operatorname{IOb}(m) \wedge \operatorname{IOb}(k) \rightarrow \exists \overline{\mathbf{y}} \forall b[\mathrm{~W}(m, b, \overline{\mathbf{x}}) \leftrightarrow \mathrm{W}(k, b, \overline{\mathbf{y}})] .
$$

From now on, we will use $\mathrm{ev}_{m}(\overline{\mathbf{x}})=\mathrm{ev}_{k}(\overline{\mathbf{y}})$ to abbreviate the subformula $\forall b[\mathrm{~W}(m, b, \overline{\mathbf{x}}) \leftrightarrow \mathrm{W}(k, b, \overline{\mathbf{y}})]$ of AxEv.

These three axioms are enough to capture the essence of special relativity. However, let us assume two more simplifying axioms.

AxSelf: Any inertial observer is stationary relative to himself:

$$
\mathrm{IOb}(m) \rightarrow \forall \overline{\mathbf{x}}\left[\mathrm{W}(m, m, \overline{\mathbf{x}}) \leftrightarrow x_{2}=\ldots=x_{d}=0\right] .
$$

Our last axiom on inertial observers is a symmetry axiom saying that they use the same units of measurement.

AxSymD: Any two inertial observers agree as to the spatial distance between two events if these two events are simultaneous for both of them; furthermore, the speed of light is 1 for all observers:

$$
\begin{aligned}
& \mathrm{IOb}(m) \wedge \mathrm{IOb}(k) \wedge x_{1}=y_{1} \wedge x_{1}^{\prime}=y_{1}^{\prime} \wedge \mathrm{ev}_{m}(\overline{\mathbf{x}})=\mathrm{ev}_{k}\left(\overline{\mathbf{x}}^{\prime}\right) \\
& \wedge \mathrm{ev}_{m}(\overline{\mathbf{y}})=\operatorname{ev}_{k}\left(\overline{\mathbf{y}}^{\prime}\right) \rightarrow \operatorname{space}^{2}(\overline{\mathbf{x}}, \overline{\mathbf{y}})=\operatorname{space}^{2}\left(\overline{\mathbf{x}}^{\prime}, \overline{\mathbf{y}}^{\prime}\right) \text { and } \\
& \mathrm{IOb}(m) \rightarrow \exists p[\mathrm{Ph}(p) \wedge \mathrm{W}(m, p, 0, \ldots, 0) \wedge \mathrm{W}(m, p, 1,1,0, \ldots, 0)] .
\end{aligned}
$$

Let us introduce an axiom system for special relativity as the collection of the five simple axioms above:

$$
\text { SpecRel }_{d}:=\mathrm{AxPh}+\mathrm{AxOField}+\mathrm{AxEv}+\mathrm{AxSelf}+\mathrm{AxSymD} .
$$

To show that the five simple axioms of SpecRel ${ }_{d}$ capture special relativity well, let us introduce the concept of worldview transformation between observers $m$ and $k$ (in symbols, $\mathbf{w}_{m k}$ ) as the binary relation on

\footnotetext{
${ }^{3}$ The supremum property (i.e., that every nonempty and bounded subset of the numbers has a least upper bound) implies the Archimedean property. So if we want to get ourselves free from the Archimedean property, we have to leave this one, too.
} 
$Q^{d}$ connecting the coordinate points where $m$ and $k$ coordinatize the same events:

$$
\mathbf{W}_{m k}\left(\overline{\mathbf{x}}, \overline{\mathbf{x}}^{\prime}\right) \stackrel{\text { def }}{\Longleftrightarrow} \forall b\left[\mathrm{~W}(m, b, \overline{\mathbf{x}}) \leftrightarrow \mathrm{W}\left(k, b, \overline{\mathbf{x}}^{\prime}\right)\right] .
$$

Map $P: Q^{d} \rightarrow Q^{d}$ is called a Poincaré transformation iff it is an affine bijection having the following property

$$
\operatorname{time}(\overline{\mathbf{x}}, \overline{\mathbf{y}})^{2}-\operatorname{space}^{2}(\overline{\mathbf{x}}, \overline{\mathbf{y}})=\operatorname{time}\left(\overline{\mathbf{x}}^{\prime}, \overline{\mathbf{y}}^{\prime}\right)^{2}-\operatorname{space}^{2}\left(\overline{\mathbf{x}}^{\prime}, \overline{\mathbf{y}}^{\prime}\right)
$$

for all $\overline{\mathbf{x}}, \overline{\mathbf{y}}, \overline{\mathbf{x}}^{\prime}, \overline{\mathbf{y}}^{\prime} \in Q^{d}$ for which $P(\overline{\mathbf{x}})=\overline{\mathbf{x}}^{\prime}$ and $P(\overline{\mathbf{y}})=\overline{\mathbf{y}}^{\prime}$.

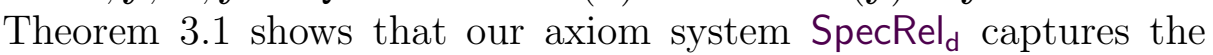
kinematics of special relativity since it implies that the worldview transformations between inertial observers are Poincaré transformations.

Theorem 3.1. Let $d \geq 3$. Assume SpecRel $d$. Then $\mathrm{w}_{m k}$ is a Poincaré transformation if $m$ and $k$ are inertial observers.

For the proof of Theorem 3.1, see 4]. For a similar result over Euclidean fields, see, e.g., [2, Thms. 1.4 \& 1.2], [3, Thm. 11.10], [17, Thm.3.1.4].

Let us now introduce a further auxiliary axiom about the possibility of motion of inertial observers.

AxThExp: Inertial observers can move along any straight line with any speed less than the speed of light:

$$
\begin{aligned}
\exists h \operatorname{IOb}(h) \wedge(\operatorname{IOb}(m) \wedge & \operatorname{space}^{2}(\overline{\mathbf{x}}, \overline{\mathbf{y}})<\operatorname{time}(\overline{\mathbf{x}}, \overline{\mathbf{y}})^{2} \\
\rightarrow & \rightarrow k[\operatorname{IOb}(k) \wedge \mathrm{W}(m, k, \overline{\mathbf{x}}) \wedge \mathrm{W}(m, k, \overline{\mathbf{y}})]) .
\end{aligned}
$$

Theorem 3.2 below shows that axiom AxThExp implies that positive numbers have square roots if SpecRel ${ }_{d}$ is assumed.

Theorem 3.2. If $d \geq 3$, then

$$
\operatorname{Num}\left(\text { SpecRel }_{d}+\text { AxThExp }\right)=\{\mathfrak{Q}: \mathfrak{Q} \text { is a Euclidean field }\} .
$$

Remark 3.3. Axiom AxThExp cannot be omitted from Theorem 3.2 since SpecRel ${ }_{d}$ has a model over every ordered field, i.e., for all $d \geq 2$,

$$
\operatorname{Num}\left(\text { SpecRel }_{d}\right)=\{\mathfrak{Q}: \mathfrak{Q} \text { is an ordered field }\}
$$

for all $d \geq 2$. Moreover, SpecRel S $_{d}$ also has non trivial models in which there are several observers moving relative to each other. We conjecture that there is a model of SpecRel $\left.\right|_{d}$ over every ordered field such that the possible speeds of observers are dense in interval $[0,1]$, see Conjecture 3.6 on p.7.

Since our measurements have only finite accuracy, it is natural to assume AxThExp only approximately. 
AxThExp ${ }^{-}$Inertial observers can move roughly with any speed less than the speed of light roughly in any direction:

$$
\begin{aligned}
& \exists h \operatorname{IOb}(h) \wedge(\operatorname{IOb}(m) \wedge \varepsilon>0 \wedge v_{2}^{2}+\ldots+v_{d}^{2}<1 \wedge v_{1}=1 \\
& \rightarrow \exists \overline{\mathbf{w}}\left[\left(w_{1}-v_{1}\right)^{2}+\ldots+\left(w_{d}-v_{d}\right)^{2}<\varepsilon \wedge \forall \overline{\mathbf{x}} \overline{\mathbf{y}} \exists \lambda(\overline{\mathbf{x}}-\overline{\mathbf{y}}=\lambda \overline{\mathbf{w}}\right. \\
&\rightarrow \exists k[\operatorname{IOb}(m) \wedge \mathrm{W}(m, k, \overline{\mathbf{y}}) \wedge \mathrm{W}(m, k, \overline{\mathbf{y}})])]) .
\end{aligned}
$$

By Theorem 3.4, a model of SpecRel ${ }_{d}+$ AxThExp $^{-}$has a model over the field of rational numbers in any dimension. We use the notation $\mathfrak{Q} \in \operatorname{Num}(\mathrm{Th})$ for algebraic structure $\mathfrak{Q}$ the same way as the model theoretic notation $\mathfrak{Q} \in \operatorname{Mod}($ AxField $)$, e.g., $\mathbb{Q} \in N u m(T h)$ means that $\mathbb{Q}$, the field of rational numbers, can be the structure of quantities in theory Th.

Theorem 3.4. For all $d \geq 2$,

$$
\mathbb{Q} \in \operatorname{Num}\left(\text { SpecRel }_{\mathrm{d}}+\mathrm{AxThExp}^{-}\right) .
$$

For the proof of Theorem 3.4, see Section 4,

An ordered field is called Archimedean field iff for all $a$, there is a natural number $n$ such that

$$
a<\underbrace{1+\ldots+1}_{n}
$$

holds. By Pickert-Hion Theorem, every Archimedean field is isomorphic to a subfield of the field of real numbers, see, e.g., [9, §VIII], [10, C.44.2]. Consequently, the field of rational numbers is dense in any Archimedean field since it is dense in the field of real numbers. Therefore, the following is a corollary of Theorem 3.4.

Corollary 3.5. For all $d \geq 2$,

$\{\mathfrak{Q}: \mathfrak{Q}$ is an Archimedean field $\} \varsubsetneqq N u m\left(\right.$ SpecRel $_{d}+$ AxThExp $\left.^{-}\right)$.

The question "exactly which ordered fields can be the quantity structures of theory SpecReld + AxThExp-?" is open. By LövenheimSkolem Theorem it is clear that Num $\left(\right.$ SpecRel $_{d}+$ AxThExp $\left.^{-}\right)$cannot be the class of Archimedean fields since it has elements of arbitrarily large cardinality while an Archimedean field has at most the cardinality of continuum since Archimedean fields are subsets of the field of real numbers by Pickert-Hion Theorem. We conjecture that there is a model of SpecRel $d+$ AxThExp ${ }^{-}$over every ordered field in any dimension, i.e.:

Conjecture 3.6. For all $d \geq 2$,

$\operatorname{Num}\left(\right.$ SpecRel $_{d}+$ AxThExp $\left.^{-}\right)=\{\mathfrak{Q}: \mathfrak{Q}$ is an ordered field $\}$. 


\section{Proof of Theorem 3.4}

In this section, we are going to prove our main result. To do so, let us recall some concepts and theorems from the literature. The following theorem is well-known, see, e.g., [15, Thm.2.1].

Theorem 4.1. The unit sphere of $\mathbb{R}^{n}$ has a dense set of points with rational coordinates.

The Euclidean length of $\overline{\mathbf{x}} \in Q^{n}$ if $n \geq 1$ is defined as:

$$
|\overline{\mathbf{x}}|:=\sqrt{x_{1}^{2}+\cdots+x_{n}^{2}} .
$$

Let us recall that the norm of linear map $A: \mathbb{R}^{d} \rightarrow \mathbb{R}^{d}$, in symbols $\|A\|$, is defined as follows:

$$
\|A\|:=\max \left\{|A \overline{\mathbf{x}}|: \overline{\mathbf{x}} \in \mathbb{R}^{d} \text { and }|\overline{\mathbf{x}}|=1\right\} .
$$

Linear bijection $A$ is called orthogonal transformation if it preserves the Euclidean distance.

Theorem 4.1 implies Theorem 4.2, see [15, Thm.3.1].

Theorem 4.2. For all orthogonal transformation $T: \mathbb{R}^{n} \rightarrow \mathbb{R}^{n}$ and any $\varepsilon>0$, there is a orthogonal transformaion $A: \mathbb{Q}^{n} \rightarrow \mathbb{Q}^{n}$ such that $\|T-A\|<\varepsilon$.

Using Theorem 4.2, let us prove that its statement also holds for Poincaré transformations.

Theorem 4.3. For every Poincaré transformation $L: \mathbb{R}^{d} \rightarrow \mathbb{R}^{d}$ and positive real number $\varepsilon$, there is a Poincaré transformation $L^{*}: \mathbb{Q}^{d} \rightarrow \mathbb{Q}^{d}$ such that $\left\|L-L^{*}\right\|<\varepsilon$.

We are going to prove Theorem 4.3 by using the fact that every Poincaré transformation is a composition of a Lorentz boost and two orthogonal transformations. Lorentz boost corresponding to velocity $v \in[0,1)$, in symbols $B_{v}$, is defined as the following linear map:

$$
B_{v} \overline{\mathbf{x}}=\left\langle\frac{x_{1}-v x_{2}}{\sqrt{1-v^{2}}}, \frac{x_{2}-v x_{1}}{\sqrt{1-v^{2}}}, x_{3}, \ldots, x_{d}\right\rangle \quad \text { for all } \overline{\mathbf{x}} \in Q^{d} .
$$

Lemma 4.4. For all Lorentz boost $B_{v}: \mathbb{R}^{d} \rightarrow \mathbb{R}^{d}$ and positive number $\varepsilon$, there is a Lorentz boost $B_{w}: \mathbb{Q}^{d} \rightarrow \mathbb{Q}^{d}$ such that $\left\|B_{v}-B_{w}\right\|<\varepsilon$.

Proof. Since, by Theorem 4.1, the set of rational points are dense in the unit circle, we have that, for all $\delta>0$ and $v \in[0,1)$, there is a $w \in \mathbb{Q} \cup[0,1)$ such that $|v-w|<\delta$ and $\sqrt{1-w^{2}} \in \mathbb{Q}$, i.e., $B_{w}$ takes rational point to rational ones. So we have to show that $\left\|B_{v}-B_{w}\right\|<\varepsilon$ if $\delta$ is small enough. Since in a finite-dimensional vector space all norms are equivalent, see [6, $\S 8.5]$, it is enough to show that the norm of $B_{v}-B_{w}$ can be less than any positive real number according to the Euclidean norm, which is 


$$
\sqrt{2\left|\frac{1}{\sqrt{1-v^{2}}}-\frac{1}{\sqrt{1-w^{2}}}\right|^{2}+2\left|\frac{v}{\sqrt{1-v^{2}}}-\frac{w}{\sqrt{1-w^{2}}}\right|^{2}} .
$$

By the continuity of functions $v \mapsto\left(1-v^{2}\right)^{-\frac{1}{2}}$ and $v \mapsto v\left(1-v^{2}\right)^{-\frac{1}{2}}$, the Euclidean norm of $B_{v}-B_{w}$ is less than any fixed positive real number if $|v-w|$ is small enough. Therefore, there is a Lorentz boost $B_{w}$ such that $B_{w}$ maps rational points to rational ones and $\left\|B_{w}-B_{v}\right\|<\varepsilon$.

Lemma 4.5. Let $A$ and $B$ be linear bijections of $\mathbb{R}^{d}$. Let $A^{\prime}$ and $B^{\prime}$ linear maps such that $\left\|A-A^{\prime}\right\|<\varepsilon_{1}$ and $\left\|B-B^{\prime}\right\|<\varepsilon_{2}$. Then $\left\|B A-B^{\prime} A^{\prime}\right\| \leq \varepsilon_{1}\|B\|+\varepsilon_{1} \varepsilon_{2}+\varepsilon_{2}\|A\|$.

Proof. First let us note that

$$
\left\|A^{\prime}\right\|=\left\|A^{\prime}-A+A\right\| \leq\left\|A^{\prime}-A\right\|+\|A\|=\varepsilon_{1}+\|A\|
$$

by the triangle inequality. Let $\overline{\mathbf{x}} \in \mathbb{R}^{d}$ such that $|\overline{\mathbf{x}}|=1$. We have to show that

$$
\left|B A \overline{\mathbf{x}}-B^{\prime} A^{\prime} \overline{\mathbf{x}}\right| \leq \varepsilon_{1}|| B\left\|+\varepsilon_{1} \varepsilon_{2}+\varepsilon_{2}|| A\right\|
$$

By the triangle inequality and the fact that $|M \overline{\mathbf{y}}| \leq|| M|| \cdot|\overline{\mathbf{y}}|$, we have

$$
\begin{aligned}
\left|B A \overline{\mathbf{x}}-B^{\prime} A^{\prime} \overline{\mathbf{x}}\right| & =\left|B A \overline{\mathbf{x}}-B A^{\prime} \overline{\mathbf{x}}+B A^{\prime} \overline{\mathbf{x}}-B^{\prime} A^{\prime} \overline{\mathbf{x}}\right| \\
& \leq\left|B A \overline{\mathbf{x}}-B A^{\prime} \overline{\mathbf{x}}\right|+\left|B A^{\prime} \overline{\mathbf{x}}-B^{\prime} A^{\prime} \overline{\mathbf{x}}\right| \\
\leq & \|B\| \cdot\left|A \overline{\mathbf{x}}-A^{\prime} \overline{\mathbf{x}}\right|+\left\|B-B^{\prime}\right\| \cdot\left|A^{\prime} \overline{\mathbf{x}}\right| \\
\leq & \|B\| \cdot\left\|A-A^{\prime}\right\|+\left\|B-B^{\prime}\right\| \cdot\left\|A^{\prime}\right\| \\
\leq & \varepsilon_{1}\|B\|+\varepsilon_{2}\left(\varepsilon_{1}+\|A\|\right)=\varepsilon_{1}\|B\|+\varepsilon_{1} \varepsilon_{2}+\varepsilon_{2}\|A\|,
\end{aligned}
$$

and this is what we wanted to prove.

Proof of Theorem 4.3. Every Poincaré transformation is a composition of a translation, a Lorentz-boost $B_{v}$ and an orthogonal transformation. Therefore, Lemmas 4.4 and 4.5 , together with Theorem 4.2 imply our statement.

Now we are going to prove Theorem 3.4, Let Id be the identity map of $\mathbb{Q}^{d}$. We denote the origin of $Q^{n}$ by $\overline{\mathbf{o}}$, i.e.,

$$
\overline{\mathbf{o}}:=\langle 0, \ldots, 0\rangle \text {. }
$$

Let the time-axis be defined as the following subset of $Q^{d}$ :

$$
\text { t-axis }:=\left\{\overline{\mathbf{x}}: x_{2}=\ldots=x_{d}=0\right\} \text {. }
$$

Let $H$ be a subset of $Q^{d}$ and let $f: Q^{d} \rightarrow Q^{d}$ be a map. The $f$-image of set $H$ is defined as:

$$
f[H]:=\{f(\overline{\mathbf{x}}): \overline{\mathbf{x}} \in H\} .
$$

The so-called worldline of body $b$ according to observer $m$ is defined as follows:

$$
\mathrm{Wl}_{m}(b):=\{\overline{\mathbf{x}}: \mathbf{W}(m, b, \overline{\mathbf{x}})\}
$$




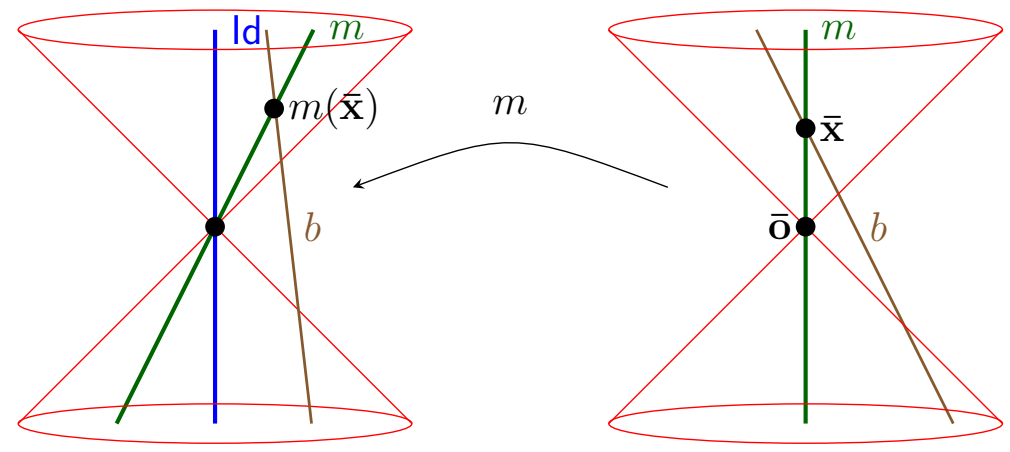

FiguRE 1. Illustration for the proof of Theorem 3.4

Proof. We are going to construct a model of SpecRel $d+$ AxThExp $^{-}$over $\mathbb{Q}$. So let $\langle Q,+, \cdot, \leq\rangle$ be the ordered field of rational numbers. Let

$$
\mathrm{Ph}:=\{l: l \text { is a line of slope } 1\},
$$

$\mathrm{IOb}:=\left\{m: m\right.$ is a Poincaré transformation from $\mathbb{Q}^{d}$ to $\left.\mathbb{Q}^{d}\right\}$,

and let $B=\mathrm{IOb} \cup \mathrm{Ph}$. First we are going to give the worldview of observer Id. Let

$$
\mathrm{W}(\mathrm{Id}, \mathrm{Id}, \overline{\mathbf{x}}) \stackrel{\text { def }}{\Longleftrightarrow} x_{2}=\ldots=x_{d}=0 ;
$$

for any other inertial observer $m$, let

$$
\mathrm{W}(\mathrm{Id}, m, \overline{\mathbf{x}}) \stackrel{\text { def }}{\Longleftrightarrow} \overline{\mathbf{x}} \in m[\text { t-axis] }
$$

and for any light signal $p \in \mathrm{Ph}$, let

$$
\mathrm{W}(\mathrm{Id}, p, \overline{\mathbf{x}}) \stackrel{\text { def }}{\Longleftrightarrow} \overline{\mathbf{x}} \in p .
$$

Now the worldview of observer Id is given. From the worldview of Id, we construct the worldview of another inertial observer $m$ as follows:

$$
\mathrm{W}(m, b, \overline{\mathbf{x}}) \stackrel{\text { def }}{\Longleftrightarrow} \mathrm{W}(\mathrm{Id}, b, m(\overline{\mathbf{x}}))
$$

for all body $b \in B$, see Figure 1.

Now we have given the model. Let us see why the axioms of SpecRel ${ }_{d}$ and $A x T_{h E x p}{ }^{-}$are valid in it.

By the above definition of $\mathbf{W}$, if $m$ and $k$ are inertial observers, then

$$
\mathrm{W}(m, k, \overline{\mathbf{x}}) \text { holds iff } m(\overline{\mathbf{x}}) \in k[\mathrm{t} \text {-axis], }
$$

and if $m \in \mathrm{IOb}$ and $p \in \mathrm{Ph}$, then

$$
\mathbf{W}(m, p, \overline{\mathbf{x}}) \text { holds iff } m(\overline{\mathbf{x}}) \in p .
$$

The worldview transformations between inertial observers $m$ and Id is $m$, i.e., $\mathbf{w}_{m \mathbf{l d}}=m$ by equation (24). Therefore, the worldview transformation between inertial observers $m$ and $k$ is $k^{-1} \circ m$, i.e.,

$$
\mathrm{w}_{m k}=k^{-1} \circ m
$$


since $\mathrm{w}_{m k}=\mathrm{w}_{\mathrm{ld} k} \circ \mathrm{w}_{m \mathrm{ld}}$ and $\mathrm{w}_{\mathrm{ld} k}=\left(\mathrm{w}_{k \mathrm{ld}}\right)^{-1}$ by the definition of the worldview transformation (5). Specially, the worldview transformations between inertial observers are Poincaré transformations in these models (as Theorem 3.1 requires it). Hence

$\mathrm{w}_{m k}$ is a bijection for all inertial observers $m$ and $k$.

Axiom $\mathrm{AxPh}$ is valid for observer Id by the definition of $\mathrm{Ph}$ and that of his worldview. It is also clear that the speed of light is 1 for observer Id. Axiom AxPh is valid for the other observers since Poincaré transformations take lines of slope one to lines of slope one. This also show that the speed of light is 1 according to every inertial observer, which is the second half of AxSymD.

Axiom AxOField is valid in this model since $\mathbb{Q}$ is an ordered field. Axiom AxEv is valid in this model since Poincaré transformations are bijections. Axiom AxSelf is valid in this model since

$$
\begin{aligned}
\mathrm{W}(m, m, \overline{\mathbf{x}}) \stackrel{(25)}{\Longleftrightarrow} m(\overline{\mathbf{x}}) \in m[\mathrm{t} \text {-axis }] & \\
& \stackrel{(20)}{\Longleftrightarrow} \overline{\mathbf{x}} \in \mathrm{t} \text {-axis } \stackrel{(16)}{\Longleftrightarrow} x_{2}=\ldots=x_{d}=0 .
\end{aligned}
$$

Any Poincaré transformation $P$ preserves the spatial distance of points $\overline{\mathbf{x}}, \overline{\mathbf{y}}$ for which $x_{1}=y_{1}$ and $P(\overline{\mathbf{x}})_{1}=P(\overline{\mathbf{y}})_{1}$. Therefore, inertial observers agree as to the spatial distance between two events if these two events are simultaneous for both of them. We have already shown that the speed of light is 1 for each inertial observers in this model. Hence axiom AxSymD is also valid in this model.

Now we are going to show that $A x \mathrm{ThExp}^{-}$is valid in this model. The $\exists h \operatorname{IOb}(h)$ part of axiom AxThExp is valid, since there are Poincaré transformations (e.g., Id is one). To show that the rest of axiom AxThExp ${ }^{-}$is valid, let $m$ be an inertial observer and let us fix an $\varepsilon>0$ and a $\overline{\mathbf{v}} \in \mathbb{Q}^{d}$ for which $v_{2}^{2}+\ldots v_{d}^{2}<1$ and $v_{1}=1$. Let $\overline{\mathbf{1}}$ be vector $\langle 1,0, \ldots, 0\rangle$. Let $L$ be a Lorentz transformation (i.e., linear Poincaré transformation) for which

$$
\overline{\mathbf{v}}=\frac{L(\overline{\mathbf{1}})}{L(\overline{\mathbf{1}})_{1}}
$$

Let $0<\delta<1$ be such that

$$
\begin{gathered}
\delta<\frac{\varepsilon L(\overline{\mathbf{1}})_{1}}{2} \quad \text { and } \\
\left|\frac{1}{L(\overline{\mathbf{1}})_{1}}-\frac{1}{x}\right|<\frac{\varepsilon}{2(\|L\|+1)}
\end{gathered}
$$

for any $x$ for which $\left|L(\overline{\mathbf{1}})_{1}-x\right|<\delta$. By Theorem 4.3, there is a Lorentz transformation $L^{*}$ which takes rational points to rational ones and $\left\|L-L^{*}\right\|<\delta$. Then

$$
\left|L(\overline{\mathbf{1}})-L^{*}(\overline{\mathbf{1}})\right|<\delta
$$


since $|\overline{\mathbf{1}}|=1$. We have $\left|L(\overline{\mathbf{1}})_{1}-L^{*}(\overline{\mathbf{1}})_{1}\right|<\delta$ since $\left|x_{1}\right|<|\overline{\mathbf{x}}|$ for all $\overline{\mathbf{x}} \in \mathbb{Q}^{d}$. By triangle inequality, we also have

$$
\left|L^{*}(\overline{\mathbf{1}})\right| \leq\left|L^{*}(\overline{\mathbf{1}})-L(\overline{\mathbf{1}})\right|+|L(\overline{\mathbf{1}})| \leq \delta+\|L\| \leq 1+\|L\| .
$$

Let

$$
\overline{\mathbf{w}}:=\frac{L^{*}(\overline{\mathbf{1}})}{L^{*}(\overline{\mathbf{1}})_{1}}
$$

By triangle inequality, we have

$$
\begin{aligned}
& |\overline{\mathbf{v}}-\overline{\mathbf{w}}| \underset{(30)}{\stackrel{(35)}{=}}\left|\frac{L(\overline{\mathbf{1}})}{L(\overline{\mathbf{1}})_{1}}-\frac{L^{*}(\overline{\mathbf{1}})}{L^{*}(\overline{\mathbf{1}})_{1}}\right| \\
& =\left|\frac{L(\overline{\mathbf{1}})}{L(\overline{\mathbf{1}})_{1}}-\frac{L^{*}(\overline{\mathbf{1}})}{L(\overline{\mathbf{1}})_{1}}+\frac{L^{*}(\overline{\mathbf{1}})}{L(\overline{\mathbf{1}})_{1}}-\frac{L^{*}(\overline{\mathbf{1}})}{L^{*}(\overline{\mathbf{1}})_{1}}\right| \\
& \leq\left|\frac{L(\overline{\mathbf{1}})}{L(\overline{\mathbf{1}})_{1}}-\frac{L^{*}(\overline{\mathbf{1}})}{L(\overline{\mathbf{1}})_{1}}\right|+\left|L^{*}(\overline{\mathbf{1}})\right|\left|\frac{1}{L(\overline{\mathbf{1}})_{1}}-\frac{1}{L^{*}(\overline{\mathbf{1}})_{1}}\right| \\
& \underset{(\overline{34})}{\leq} \frac{1}{L(\overline{\mathbf{1}})_{1}}\left|L(\overline{\mathbf{1}})-L^{*}(\overline{\mathbf{1}})\right|+(1+\|L\|)\left|\frac{1}{L(\overline{\mathbf{1}})_{1}}-\frac{1}{L^{*}(\overline{\mathbf{1}})_{1}}\right| \\
& \underset{(32)}{\stackrel{(33)}{<}} \frac{\delta}{L(\overline{\mathbf{1}})_{1}}+\frac{\varepsilon}{2} \underset{(31)}{<} \varepsilon
\end{aligned}
$$

Let $\overline{\mathbf{x}}, \overline{\mathbf{y}} \in \mathbb{Q}^{d}$ such that there is a $\lambda \in \mathbb{Q}$ such that $\overline{\mathbf{y}}-\overline{\mathbf{x}}=\lambda \overline{\mathbf{w}}$. To finish the proof of $A x T^{2} E x p^{-}$, we have to show that there is an inertial observer $k$ such that $\mathbf{W}(m, k, \overline{\mathbf{x}})$ and $\mathbf{W}(m, k, \overline{\mathbf{y}})$, i.e., $\overline{\mathbf{x}}, \overline{\mathbf{y}} \in \mathrm{wl}_{m}(k)$. Let $P^{*}=L^{*}+\overline{\mathbf{x}} . P^{*}$ is a Poincaré transformation taking rational points to rational ones. Therefore, there is an inertial observer $k$ such that $\mathbf{w}_{k m}=$ $P^{*}$. Since $\mathrm{wl}_{m}(k)=\mathrm{w}_{k m}\left[\mathrm{t}\right.$-axis], we have that $\mathrm{w}_{k m}(\overline{\mathbf{o}})=\overline{\mathbf{x}} \in \mathrm{wl}_{m}(k)$ and that $\overline{\mathbf{y}}=a L^{*}(\overline{\mathbf{1}})+\overline{\mathbf{x}}=\mathrm{w}_{k m}(a \overline{\mathbf{1}}) \in \mathrm{wl}_{m}(k)$, where $a=\lambda / L^{*}(\overline{\mathbf{1}})_{1}$. This shows that AxThExp ${ }^{-}$is also valid in our model.

\section{ACKNOWLEDGMENTS}

This research is supported by the Hungarian Scientific Research Fund for basic research grants No. T81188 and No. PD84093, as well as by a Bolyai grant for J. X. Madarász.

\section{REFERENCES}

[1] H. Andréka, J. X. Madarász, and I. Németi, with contributions from: A. Andai, G. Sági, I. Sain, and Cs. Töke. On the logical structure of relativity theories. Research report, Alfréd Rényi Institute of Mathematics, Hungar. Acad. Sci., Budapest, 2002. http://www.math-inst.hu/pub/algebraic-logic/Contents.html.

[2] H. Andréka, J. X. Madarász, and I. Németi. Logical axiomatizations of spacetime. Samples from the literature. In A. Prékopa and E. Molnár, editors, NonEuclidean geometries, pages 155-185. Springer-Verlag, New York, 2006.

[3] H. Andréka, J. X. Madarász, and I. Németi. Logic of space-time and relativity theory. In M. Aiello, I. Pratt-Hartmann, and J. van Benthem, editors, Handbook of spatial logics, pages 607-711. Springer-Verlag, Dordrecht, 2007. 
[4] H. Andréka, J. X. Madarász, I. Németi, and G. Székely. What are the numbers in which spacetime?, 2012. arXiv:1204.1350v1 [gr-qc].

[5] J. Ax. The elementary foundations of spacetime. Found. Phys., 8(7-8):507-546, 1978.

[6] G. Bachman and L. Narici. Functional analysis. Dover Publications Inc., Mineola, NY, 2000. Reprint of the 1966 original.

[7] C. C. Chang and H. J. Keisler. Model theory. North-Holland Publishing Co., Amsterdam, 1990.

[8] H. B. Enderton. A mathematical introduction to logic. Academic Press, New York, 1972.

[9] L. Fuchs. Partially ordered algebraic systems. Pergamon Press, Oxford, 1963.

[10] A. V. Mikhalev and G. F. Pilz, editors. The concise handbook of algebra. Kluwer Academic Publishers, Dordrecht, 2002.

[11] E. E. Rosinger. Two essays on the archimedean versus non-archimedean debate, 2008. arXiv:0809.4509v3.

[12] E. E. Rosinger. Special relativity in reduced power algebras, 2009. arXiv:0903.0296v1.

[13] E. E. Rosinger. Cosmic contact to be, or not to be archimedean. Prespacetime Journal, 2(2):234-248, 2011.

[14] E. E. Rosinger. How far should the principle of relativity go? Prespacetime Journal, 2(2):249-264, 2011.

[15] E. Schmutz. Rational points on the unit sphere. Central European Journal of Mathematics, 6(3):482-487, 2008.

[16] M. Stannett. Computing the appearance of physical reality. Appl. Math. Comput., in press, 2011.

[17] G. Székely. First-Order Logic Investigation of Relativity Theory with an Emphasis on Accelerated Observers. PhD thesis, Eötvös Loránd Univ., Budapest, 2009.

[18] A. Tarski. What is elementary geometry? In The axiomatic method. With special reference to geometry and physics. Proceedings of an International Symposium held at the Univ. of Calif., Berkeley, Dec. 26, 1957-Jan. 4, 1958 (edited by L. Henkin, P. Suppes and A. Tarski), pages 16-29, Amsterdam, 1959. NorthHolland Publishing Co.

[19] J. Väänänen. Second-order logic and foundations of mathematics. Bull. Symbolic Logic, 7(4):504-520, 2001.

[20] J. Woleński. First-order logic: (philosophical) pro and contra. In V. F. Hendricks et al., editors, First-Order Logic Revisited, pages 369-398. Logos Verlag, Berlin, 2004.

Alfréd Rényi Institute of Mathematics, Hungarian Academy of Sciences, ReÁltanoda utca 13-15, H-1053, Budapest, Hungary

E-mail address: \{madarasz.judit, szekely.gergely\}@renyi.mta.hu 